“C 2018 IEEE. Personal use of this material is permitted. Permission from IEEE must be obtained for all other uses, in any current or future media, including reprinting/republishing this material for advertising or promotional purposes, creating new collective works, for resale or redistribution to servers or lists, or reuse of any copyrighted component of this work in other works."

According to IEE Policy*, in the next pages the accepted version of the paper is reproduced.

- see https://ieeeauthorcenter.ieee.org/publishing-ethics/guidelines-and-policies/policy-posting-yourarticle/ 


\title{
Modelling and Simulation of AC Railway Electric Supply Lines Including Ground Return
}

\author{
Massimo Ceraolo - University of Pisa, \\ Largo Lazzarino 56122 Pisa Italy voice: +39 0502217305 \\ massimo.ceraolo@unipi.it
}

\begin{abstract}
Today's AC railway feeding systems are composed by several conductors with various connections to each other. The return current is shared between rails, ground wires connected to rails, ground; current flows to ground are determined by rail-to-ground distributed conductance. This paper gives formulas to deal with all these complexities, both for power quality and power frequency studies, integrating and combining previous studies which cover only part of them.

Especially new in this paper is a technique to include in the computation currents dispersed into soil through ballast.

It also illustrates a way to implement easily all the proposed formulas using a specific, open and flexible simulation language, the Modelica language. An application of the proposed formulas and related simplifications is illustrated.
\end{abstract}

Keywords-Railway line; Ballast, Carson model; Electrified Transportation, Modelica; Simulation, Time-domain; Frequency domain.

\section{INTRODUCTION}

Worldwide, several kinds of feeding systems for railways are used, either DC (e.g. $1500 \mathrm{~V}, 3000 \mathrm{~V}), \mathrm{AC}$ at a special frequency $(16.7 \mathrm{~Hz}, 25 \mathrm{~Hz}), \mathrm{AC}$ at ordinary frequency $(50,60$ $\mathrm{Hz}$ ).

However, for high speed lines AC dominates because it more easily allows creating the higher voltage needed for the high power requested by high speed transit.

Mathematical description of $\mathrm{AC}$ lines is much more complex than that of DC's for a number of factors:

- magnetic and electric field interaction between live conductors

- interaction with additional conductors such as ground wires

- current flowing into ground determined by ballast's distributed conductance as well as electromagnetic interaction with live conductors.

Modelling AC railway lines may be necessary for different purposes, the main of which are:

- Power quality analysis. In this case some "photographs" of the system operation are taken, with the train being in a fixed position, and harmonic interaction between all the conductors is studied e.g. to evaluate the induced voltage on external wires

- Power Frequency analysis. For this analysis trains are followed during their trips for several seconds, minutes or more; in this case the main objective of the study is to evaluate power-frequency RMS components of voltages and currents, as well as active and reactive powers. For these studies on one hand modelling the electric supply system is simpler since there is no need of higher frequency component analysis, on the other much faster simulation is required to be able to make long run analyses.

A literature survey shows that good articles exist dealing with some of these aspects, but none of them considers all the phenomena listed above simultaneously.

Paper [1] is historical, since has laid a theory which has been used for nearly a century for modelling the effects of ground in transmission line simulations. However, this paper uses CGS formulas, which are structurally different from SI ones and are difficult to read today. Carson's theory has some limitations: it neglects soil's displacement currents and considers the soil as having a uniform resistivity; these hypotheses are held in this paper, although some specialist papers have proposed formulations to overcome them [19]. This is reasonable for the objective of this paper, which is to create techniques and models to perform power frequency and power quality studies: both require to consider frequencies of at most a few $\mathrm{kHz}$.

Paper [20] shows formulas to reduce the size of the transmission line matrices; in this paper they are mentioned and used, and their use and limitations discussed.

Hill and others have studied thoroughly rail resistances and internal inductances $[3,4,6]$. However they did not consider rail related external inductances, for which common formulas related to cylindrical conductors cannot be applied due to the different shape of magnetic field produced. This issue has been analysed by the author in a recent paper which he coauthored [25] and the results used here.

Finally, the literature analysis performed has not revealed recent papers considering the inclusion in the ground return model the distributed rail-to-ground conductance. Papers [12, 14] consider them but [12] refers to DC lines only and is in Italian, while [14], deals with this issue marginally, and is published in a basically national-wide journal.

In this paper, a rather detailed analysis of this issue, with reference to AC lines, is enclosed.

\section{PROBLEM FORMULATION}

To formulate the problem we start from a realistic example, taken from paper [20], representing a state -of-the art AC system, normally called $2 \times 25 \mathrm{kV}$ and used by several countries, among which Japan, France, Italy, and Iran [21].

The considered system contains two tracks; the complete set of conductors is graphically represented in fig. 1 .

The shown conductors are those referred to in table I: 
Table I. Conductor names and symbols.

\begin{tabular}{|l|l|l|l|}
\hline Type & Name & Type & Name \\
\hline FD & Feeder & $\mathrm{CW}$ & Contact Wire \\
\hline $\mathrm{M}$ & Messenger & $\mathrm{Ra}, \mathrm{Rb}$ & Rails \\
\hline EW & Earth wire & $\mathrm{GW}$ & Ground wire \\
\hline RW & Return Wire & & \\
\hline
\end{tabular}

Optionally, additional conductors external to the traction line may be included in the conductor bundle, so that the induced voltage on them can be also computed, to evaluate electromagnetic interference.

For the analysis of this system not only the conductors (including rails) shown in figure 1 must be considered, but also the ground. In fact, the traction line may be explicitly grounded at sending and receiving ends, and some leakage current also abandons the rails to enter the ground, because distributed linear conductance exists, whose value can vary widely. Values between 0.02 and $20 \mathrm{~S} / \mathrm{km}$ are considered by [7]. Paper [10] shows that, depending on country, distributed conductance values can range from $0.01 \mathrm{~S} / \mathrm{km}$ up to $40 \mathrm{~S} / \mathrm{km}$. The conductance values are influenced by the presence of buried earthing conductor running along the line, frequently connected to rails.

The value of this distributed conductance for high speed lines cannot be too small, because of the high currents flowing through rails, which can easily reach a few hundred amperes. This justifies to have considered in this paper a smaller range than the one mentioned in [10], i.e. 0.1-10 S/km. O FD1

FD O

O M1 M O

O RW1

O cW1

$\mathrm{CW} O$

O RW
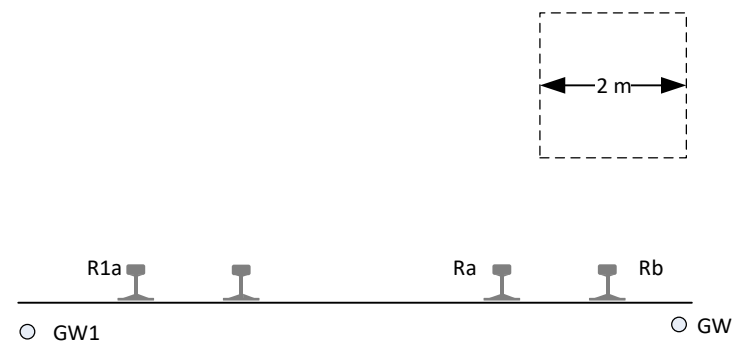

Figure 1. A physical line example.

The conductor distances do not remain constant along the railways abscissa, since the suspended conductors take a catenary shape. However, theoretical and practical analysis has shown that, at least for frequencies up to a few $\mathrm{kHz}$, considered in this study, we can take the conductors as being straight lines, located in the transverse plane (that of figure 1) at their average height.

In a longitudinal view, the system in figure 1 could be represented as in figure 2 , in which $u_{\mathrm{kj}}$ and $i_{\mathrm{kj}}$ are time functions and the corresponding relations are differentialalgebraic equations.

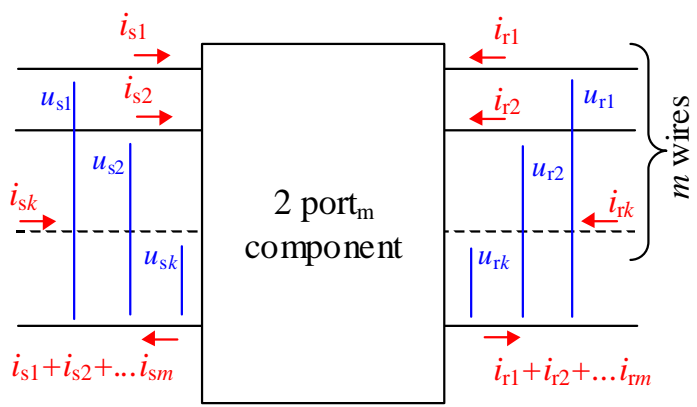

Figure. 2. A linear multi-conductor transmission line (MTL) can be modelled as a two-port component.

If these relations are linear and the system operates with sine quantities, at a given frequency, these can be expressed in terms of phasors, and be written as:

$$
\left\{\begin{array}{l}
\boldsymbol{U}_{\boldsymbol{s}}=\boldsymbol{A} \boldsymbol{U}_{\boldsymbol{r}}+\boldsymbol{B} \boldsymbol{I}_{r} \\
\boldsymbol{I}_{s}=\boldsymbol{C} \boldsymbol{U}_{r}+\boldsymbol{D I} \boldsymbol{I}_{r}
\end{array}\right.
$$

Where $\boldsymbol{A}, \boldsymbol{B}, \boldsymbol{C}, \boldsymbol{D}$ are matrices made of complex numbers.

To find these matrices we make the two-port $\mathrm{m}_{\mathrm{m}}$ component of figure 2 "transparent", as in figure 3 , in which the physical nature of the two-port $\mathrm{m}_{\mathrm{m}}$ component of figure 2 is shown, i.e. we have horizontal wires which are a given height over ground, among which (and among them and ground) electrical and magnetic coupling exists and must be adequately modelled. The uppercase letters for voltages and currents now indicate complex numbers representing phasors at the considered frequency.

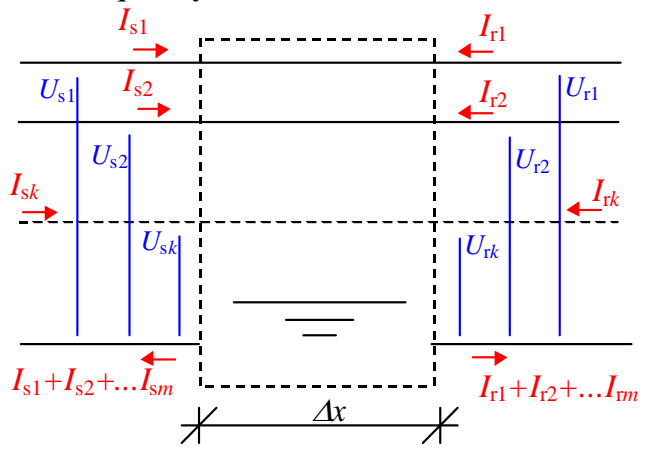

Figure 3. Specific two-port structure of a MTL.

If $\Delta x$ is infinitesimal, i.e. it is $\mathrm{d} x$, the telegrapher's equation, for sine waves and a Multi-Conductor Line (MTL), can be written as:

$$
-\frac{d \boldsymbol{U}}{d x}=\boldsymbol{Z}^{\prime} \boldsymbol{I} \quad-\frac{d \boldsymbol{I}}{d x}=\boldsymbol{Y}^{\prime} \boldsymbol{U}
$$

Were $\boldsymbol{U}=\left[U_{1}, U_{1}, \ldots U_{\mathrm{m}}\right], \boldsymbol{I}=\left[I_{1}, I_{2}, I_{\mathrm{m}}\right]$ and the difference between sections $s$ (left, or sending end) and $r$ (right, or receiving end) vanishes being the length infinitesimal. $Z$ ' and $Y^{\prime}$ are impedance and admittance matrices per unit length. Eq. (2) is derived in textbooks dealing with MTL's, such as [9 or 13]. $Z$ ' can be derived from Carson's theory in case no rail-toground dispersion is considered; the corresponding formulas are reported in Appendix A. Formulas for $\boldsymbol{Y}^{\prime}$ also in Appendix A.

When current dispersion to ground is not negligible, to the author's knowledge, no closed formulas exist for $\boldsymbol{Z}$ ' and $Y^{\prime}$, A 
correct simulation can be made using cascaded PI's, as detailed in the following section.

Equation (2) can be rewritten as:

$$
\frac{d^{2} \boldsymbol{U}}{d x^{2}}=\boldsymbol{Z}^{\prime} \boldsymbol{Y}^{\prime} \boldsymbol{I} \quad \frac{d^{2} \boldsymbol{I}}{d x^{2}}=\boldsymbol{Z}^{\prime} \boldsymbol{Y}^{\prime} \boldsymbol{U}
$$

which is solved in power systems textbooks, and in particular in [13], in its chapter 6. This gives rise to a formulation of $\boldsymbol{A}$, $B, C, D$ constants.

If we limit to the case to $m=1$, when $\boldsymbol{Z}$ ' and $\boldsymbol{Y}^{\prime}$ are known, for a given radian frequency $\omega$ matrices $\boldsymbol{A}, \boldsymbol{B}, \boldsymbol{C}, \boldsymbol{D}$ are scalar and are expressed by the following expressions, $L$ being is the two-port component length [see for instance 9 pag. 196-197]:

$$
\begin{gathered}
A=\cosh K L \quad B=Z_{c} \sinh K L \\
C=\frac{\sinh K L}{Z_{c}} \quad D=\cosh K L
\end{gathered}
$$

where $Z_{c}=\sqrt{Z^{\prime} / Y^{\prime}}, K=\sqrt{Z^{\prime} Y^{\prime}}$.

This gives rise to an equivalent circuit representation of the two-port component as in figure $4 \mathrm{a}$ (horizontal rectangles are impedances, vertical admittances):
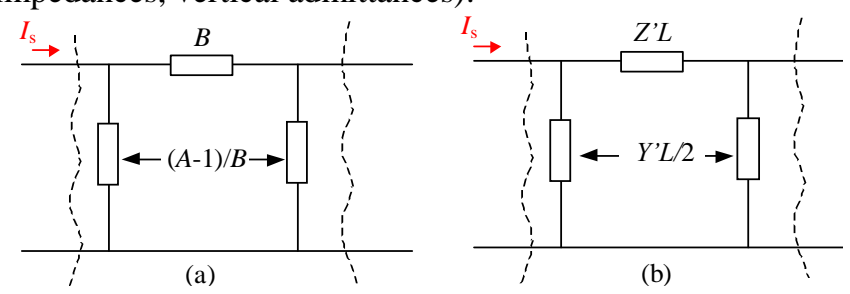

Figure 4. PI model of an AC single wire under sine voltages and currents. Left: generic, right, "nominal pi".

The curved-dashed lines are kept to remind that this circuit cannot be used to evaluate cross voltage between sending and receiving end of the line (cfr. App. A of 17).

If we consider a lossless line, $K$ is equal to $2 \pi$ times the inverse of the wavelength, which in turn can be computed with good approximation as the wavelength of electromagnetic wave in empty space. So, for instance at $50 \mathrm{~Hz}$ the wavelength is $6000 \mathrm{~km}$. This implies that for railway line trunks, whose length does not overcome a few tens of $\mathrm{km}, K L$ is small in comparison with unity, and it can be assumed that $\sinh K L \cong$ $K L, \cos K L \cong 1+(K L)^{2} / 2$, so the longitudinal impedance $B \cong Z^{\prime} L$, and the transverse admittances $(A-1) / B=Y^{\prime} L / 2$ (fig. 4b).

Consequently, for lines up to a few hundred $\mathrm{km}$, the parameters of the equivalent pi circuit can be assumed proportional to the line length, which eases manual and automatic computation.

The case of railway line is very special, because of the presence of the distributed conductance between rails and soil.

In the next section we will show that the hyperbolic formulas (4) are to be considered (and can actually be used for DC lines) for railway lines with distributed conductance even for relatively short lengths.

\section{RAIL AND GROUND-RELATED ISSUES}

The presence of rail makes the computation of the supply line more complicated than the generic case considered by power engineers, and summarized in the previous section.
Several special characteristics must be tacked, among which:

1. Rails are made of ferromagnetic and nonlinear materials and are not circular in shape

2. Some current flows between rails and soil through the ballast.

As with usual power lines some current flows through the ground; however, the special geometry of the rail-feeding lines requires some specific evaluation to be done.

Point 1 is well analyzed by Hill et al, in papers [3], [4], [6]. They especially analyzed rail resistance and "inner inductance", i.e. the one due to flux flowing inside the conductor. A rapid hint on rail external inductance, which in principle cannot be computed using the formulas for cylindrical conductors due to the special shape of the magnetic field outside rails, is in [25], which promises a specific paper on this issue. The result from these studies can be summarized as in the following list (for details the reader is prompted to consult the original papers):

- the AC rail resistance is between 80 and $240 \mathrm{~m} \Omega / \mathrm{km}$

- the AC rail internal impedance is between 0.3 and 0.9 $\mathrm{mH} / \mathrm{km}$

- the external AC inductance can be computed while approximating the rail to a cylindrical conductor having as diameter the rail height.

Point 2 is very special, and not large literature exists. The following two sections analyze it.

\section{A. Distributed current dispersion to soil}

Regarding the rails to ground contact, and the corresponding distributed current to soil modelling, no large literature exists. The writer has found mainly paper [12], which is limited to DC and is in Italian, and [14], which deals with the topic marginally

All the authors consider the dispersion current trough ballast towards soil as correctly described by a distributed conductance rail-soil. This is reasonable and accepted here.

Consider first a line operating in DC. A special version of (3) can be written as follows:

$$
\frac{d^{2} U}{d x^{2}}=r g I \quad \frac{d^{2} I}{d x^{2}}=r g U
$$

where $r$ is the rail resistance per unit length (e.g. $0.1 \Omega / \mathrm{km}$ ), and $g$ the rail-to-soil distributed conductance (e.g. $0.5 \mathrm{~S} / \mathrm{km}$ ).

To move from (3), written in scalar form, to (5) we can consider figure 5 , where complex quantities are represented underlined. The two equations can be written from the circuits in figure 5: since they have the same symbolic structure, we get the same symbolic consequences. 

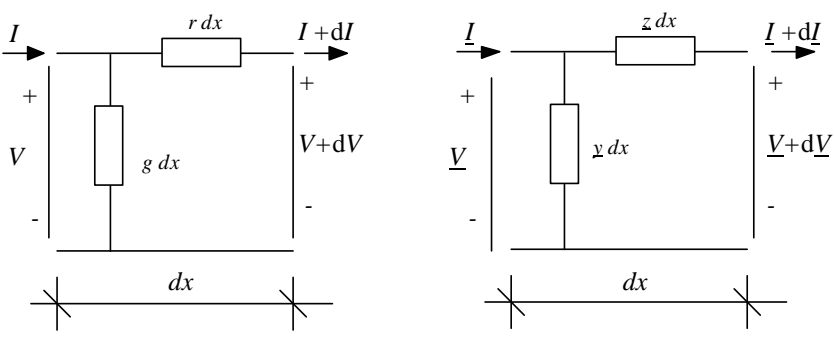

Figure 5. Structural identity of DC (left) and AC (right) circuit for a $\mathrm{d} x$-long two-wire line trunk.

Since (5) has the same structure (3) has when $m=1$ it has the same solution of it. Equations (4) can be used, substituting $Z$ ' with $r$, and $Y^{\prime}$ with $g$, and the corresponding pi-circuit of figure 4 can be still be used.

Now $K=\sqrt{r g}$. and $R_{c}=\sqrt{r / g}$. The numerical value of $1 / K$ is in the order a few tenths of $\mathrm{km}$, and therefore, the pi equivalent such as the one in figure $4 \mathrm{a}$ can be used, as per fig. $6 \mathrm{a}$, but the much simpler nominal pi (fig. $6 \mathrm{~b}$ ) can be used only for small values of $K L$, e.g. assuming a $(K L)_{\max }=0.3$ for nominal pi, which happens only for relatively small maximum lengths.
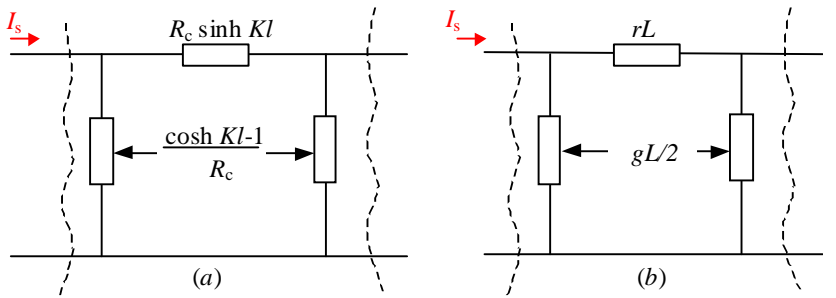

Figure 6. PI model for modelling DC current dispersion to ground Left: generic, right, "nominal pi".

From figure 6 we observe that the dispersion to ground falls well within the theory of linear AC lines, for which (1) and (3) apply: $g$ is simply the real part of $\boldsymbol{Y}^{\prime}$. However, in this case $\boldsymbol{A}$, $\boldsymbol{B}, \boldsymbol{C}, \boldsymbol{D}$ are complex matrix expressions, which are very difficult to manage and cannot be simplified for usual rail lengths the much simpler formulas such as the ones shown in figure $4 \mathrm{~b}$, because this simplified circuit now is valid only for too short lines, i.e. for lengths at most of $1 \mathrm{~km}$ or two.

\section{B. Modelling of ground return behaviour}

Consideration of ground return involves also modelling of ground behavior, since part of the train return current escapes rails and flows in soil.

Again, also in this case there are not special reasons to consider invalid the MTL model as summarized by (1) and (3). So, as per the case of conventional power lines, a separate analysis can be made for computation of longitudinal impedances (elements $\boldsymbol{Z}^{\prime}$ ) and transverse admittances (elements of $\boldsymbol{Y}^{\prime}$ ).

The longitudinal impedances with ground return can be evaluated using the theory developed by Carson a long time ago [1], valid under the hypothesis of a linear soil with uniform resistivity, and neglecting displacement currents through soil. Paper [5] shows that Carson's formulas, for uniform soil resistivity, are good enough up to $4 \mathrm{MHz}$, so they are totally acceptable for this paper's purposes.
The practical implementation of Carson's formulas is not very easy, especially if we want to start from the original Carson's paper. Therefore, for the reader's convenience, Appendix A reports these formulas in a modern way, exactly the way used in the simulation software.

In the following, numerical evaluation of the effects of distributed conductance between rails and ground and evaluation of current share between rails and ground considering Carson formulas are shown using a mathematical model built using this paper's formulas in Modelica Language $[16,18]$, taking as reference to the railway physical structure shown in figure 1.

\section{A CASE STUDY}

\section{A. Description}

In principle the formulas given in this paper should give acceptable results for simulations in the frequency domain for frequencies up to several $\mathrm{kHz}$ : they are based on Carson's formulas, which are valid in this frequency range.

However, the main focus of this paper is on power frequency modelling, to evaluate voltages, currents, active and reactive powers. Therefore all the formulas given here can be used in the frequency domain at a single frequency equal to power frequency.

One of the contributions of this paper is the evaluation of the current flowing in the rails and in the soil. Therefore, as a significant case study, we take the $2 \times 25 \mathrm{kV}$ feeding system that is very widely used for high speed lines in Europe, whose physical appearance has already been shown in figure 1, even though, to ease analysis of the results, only a single track is simulated. The physical structure of one track of figure 1 is inserted in a system containing autotransformers, the typical arrangement of a $2 \times 25 \mathrm{kV}$ system for high-speed railway feeding systems, as shown in Appendix B. In our model GW is implicitly considered, since it contributes to the distributed conductance to soil.

\section{B. Modelica Modelling}

A lot of studies have proposed simulation results obtained with proprietary or commercial software. The usage of Modelica models, instead, has the advantage that this language is standardized by the Modelica association [27] and the Modelica models can be run virtually using one of the several available Modelica-based simulation tools, either commercial or free [15].

Modelica is a language in which formulas such as those from Appendix A can be written in terms of equations, much the same we do in papers and books. All the complexities connected with numerical simulation, system reduction, dynamical step adaptation, and so on, are hidden from the final user that, except for special cases does not need to deal with them. Each track of the line in fig. 1 consists of 6 conductors (as said, GW is implicitly considered within distributed conductance to soil). Some of them, however, are frequently connected to each other creating what [20] calls a "distributed shunted" connection $\mathrm{M}$ and $\mathrm{CW}$ are continuously connected to each other, but also rails and EW and GW are frequently connected. Furthermore GW is connected to rails.

To create a simulator, MTL models of the type in figure 2, can be created whenever we have a MTL whose quantities 
vary only longitudinally. For instance, if he have a train in between a cell (figure 7), the two cell trunks can be considered upstream and downstream the train.

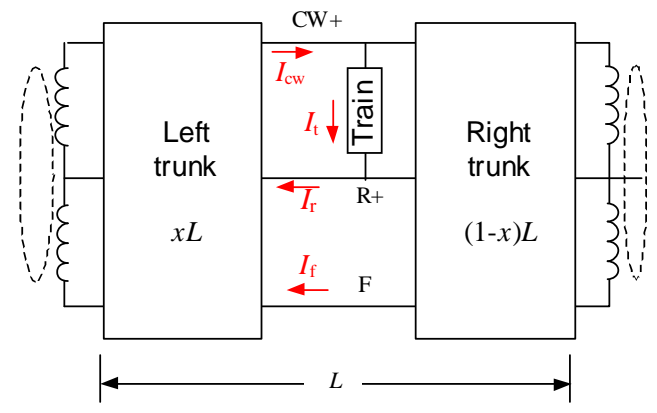

Figure 7. Simulation model using two cell trunks and equivalent conductors $\mathrm{CW}+$ and $\mathrm{R}+$.

Whatever the position $x$ of the train, at the two sides of a trunk we can consider parallel connections, e.g. between $\mathrm{CW}$ and $\mathrm{M}$, or between rails and $\mathrm{GW}$, to occur. Once this connection is made at the two trunk sides, there is no need to replicate it inside, since the trunk structure itself guarantees that at each longitudinal in-trunk position voltages of paralleled conductors remain equal to each other. So the system may be arranged in such a way that only three equivalent wires are exposed: $\mathrm{CW}^{+}$, constituted by contact wire and messenger, $\mathrm{R}^{+}$, rails and conductors in parallel with them, $\left(\mathrm{R}^{+}=\mathrm{Ra}+\mathrm{Rb}+\mathrm{RW}+\mathrm{GW}\right)$ and feeder $\mathrm{F}$.

When parallel connections are to be made, the matrixes $\boldsymbol{Z}$ ' and $\boldsymbol{Y}^{\prime}$ of (3) can be reduced in size and transformed. Formulas and algorithms to make these transformations are be for instance proposed and discussed in [20]. For instance Contact Wire can be paralleled with Messenger and the two rails each other. This on one hand reduces the matrix's sizes, on the other implies the share of current between paralleled conductors to be lost.

This matrix manipulation can be made at power frequency or harmonic frequency, given that the considered frequency is within the field of validity of the models, as discussed previously. Particularly important is the analysis in the power frequency domain, since the obtained model of rail feeding system can be used within dynamic simulations for instance to evaluate active and reactive power flows when trains are moving, accelerating, braking. Because of this, in the following sections, whenever not otherwise stated, the proposed results are obtained using a frequency of $50 \mathrm{~Hz}$.

\section{Dealing with distributed rail-soil conductance}

The issue of what to put inside each trunk is mainly related to the effects of distributed conductance towards soil.

In section III.A it has been seen that a DC line with dispersion can be modelled by a simplified PI (such as the one at figure 6b) only when $K L$ is small, say $K L<0.3$. It can be hypothesized that this result stays valid also in $\mathrm{AC}$ with a multi-conductor line, in such a way that for small values of $K L$ the pi equivalent shown in figure $4 \mathrm{~b}$ can be used.

To verify this, the current flowing in the rails, $\left(I_{\mathrm{r}}\right.$ in fig. 7$)$ can be evaluated with simulations. It is known from theory, and also mentioned in [24], pag. xi, that this current, under hypotheses typically roughly verified in practice, is approximately equal to the train current when the train is near the beginning of the cell $(x \approx 0)$, and equal to zero when $x \approx 1$. Correspondingly $I_{\mathrm{f}}$ should go from 0 to $I_{\mathrm{t}} / 2$. When the train exits the cell, then, the currents in $\mathrm{CW}^{+}$and $\mathrm{F}$ are half the train current.

During this evaluation it is convenient to introduce in left and right trunks $N$ cascaded nominal PI models. Each of the PI's will have as length $x L / N$. and $(1-x) L / N$ respectively. The higher the number, the more accurate the simulation. Inside each PI, if the inner distribution of current between actual conductors is not of interest, the above-mentioned "distributed shunting" technique to reduce the size of each PI matrices may be adopted.

Results with train as an impedance $(\cos \varphi=0.95)$, with $r$ (due to the combination of both rails) $=0.075 \Omega / \mathrm{km}$ and $g=1 \mathrm{~S} / \mathrm{km}$, are shown in figure 8 , which can be discussed as follows:

- In the upper plot rail Ra currents are shown; Rb currents are very near to them, but a slight difference exists, not shown here for brevity.

- The train current is composed by the sum or currents flowing in $\mathrm{Ra}, \mathrm{Rb}, \mathrm{RW}$. When $x \approx 0$ they are equal to each other because of the systems symmetry (not broken by current asymmetry along the line, which instead plays a role when $x>0$ ).

- The current flowing in the return path is shared between the two rails and RW. The behaviour is not linear with the train distance from the line beginning, because of the distributed conductance (becomes linear when distributed conductance is negligible).

- Moving from the usage of one PI block to eight PI blocks influences the result not negligibly. A further simulation (not reported here), however shows that the result between 8 and 12 blocks are pretty much the same. This confirms that the number of PI's to be used can be a priori computed imposing that, for each of them $\frac{K L}{N}=\sqrt{r g}(L / N)<0.3$. In the present case, in which $r=0.075 \Omega / \mathrm{km}$ and $L=12.5 \mathrm{~km}$, $g=1 \mathrm{~S} / \mathrm{km}$ requires $N>11$. If a slightly lower precision is wished, $K L / N<0.5$ can be imposed.

- The current drawn by the train varies very little during the train movement along the cell; being this current is proportional to the voltage, also the voltage is nearly constant.

As mentioned earlier, in the plots in figure 1 each PI of each trunk is variable in length during train movement.

This model, with a sufficient number of PI's considered, allows accurate evaluation of the rails voltage, which is the potential difference of rails and far earth (Carson conductor). This is shown in fig. 9. Note that the voltage values in figure are well below the safety constraints, and will stay such even at the maximum possible train currents, i.e. $500 \mathrm{~A}$. The same does not hold true for much smaller values of $g$ : re-simulating with $g=0.1 \mathrm{~S} / \mathrm{km}$ gives a rail-to-ground voltage that reaches $100 \mathrm{~V}$, which is well beyond safety limits (usually set around $50-65 \mathrm{~V})$, in the worst train position and $I_{\text {train }}=500 \mathrm{~A}$. 

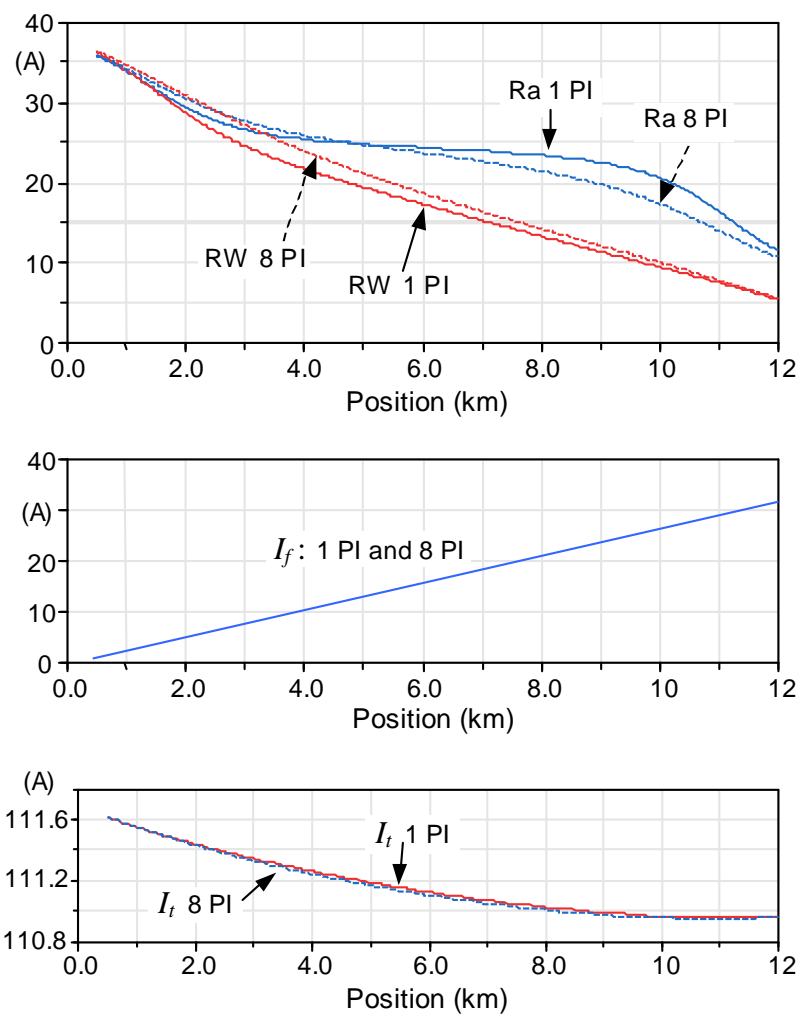

Figure 8 Currents (RMS) as a function of train position when using 1 , or 8 cascaded PI for each of the two cell trunks.

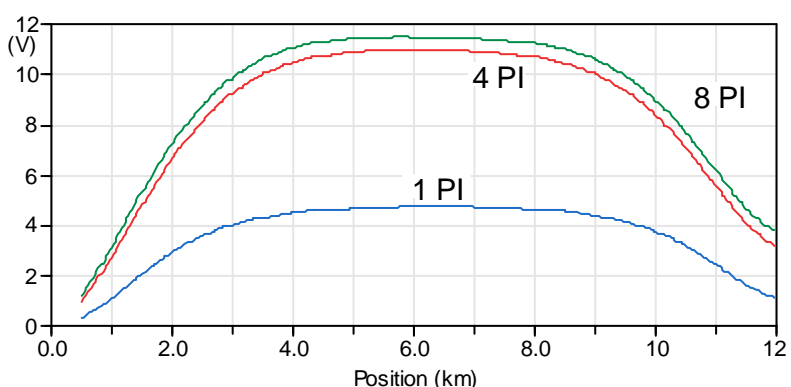

Figure 9. Rail voltages (RMS) as computed with different numbers of PI models $(g=1 \mathrm{~S} / \mathrm{km})$.

We must underline that the voltage evaluation reported in figure 9 must be considered provisional, since it comes from the proposed combination of Carson's theory and use of distributed conductance to model continuous dispersion of current to ground.

Moreover, the voltages in figure 9 are total rail-to-ground voltages; actual step voltages, which is what really matters for safety, are a fraction of them. How small this fraction is, is not evaluated here since we have only a longitudinal model of the railway line. Hovever, if the total voltage is below the $50 \mathrm{~V}$ limit, also step voltages will be safe. 9.

See sect. IV.E for further comments on the validity of figure

The typical characteristic of $2 \times 25 \mathrm{kV}$ systems is that the current in the return path should reduce linearly when the train moves along the cell, reaching zero or nearly zero when the train reaches the end of the cell ad remains null when the train occupies the subsequent cells.

This behavior constitutes the largest benefit of $2 \times 25 \mathrm{kV}$ systems, making it capable of transmitting at lower joule losses and voltage drops than the cheaper $1 \times 25 \mathrm{kV}$ counterpart. This behaviour can be easily demonstrated under the following assumptions:

- Voltage drops due to resistances negligible

- Self-inductance of the line constituted by CW and return path equal to the one of the line constituted by feeder and return path

- Idealized transformers and autotransformers, so that they are able to divide exactly in half the voltage between $\mathrm{CW}$ and feeder

- Negligible current dispersion from rails to ground.

Real-life lines are rather far from these assumptions, but actual geometries are built so that that useful behavior is approached.

The effect of distributed conductance value $g$ on returnpath current has been evaluated in simulations using always a sufficient number of cascaded PIs, some results of which are shown in figure 10 . We see that when $g=0.1 \mathrm{~S} / \mathrm{km}$ we have a return-path current nearly linearly decreasing (i.e. similar to what expected fro $2 \times 25 \mathrm{kV}$ systems according to the usual analysis), even though the return path current when the train at the end of the cell is not exactly zero. This behavior says that the line geometry is able to reproduce, with its complexity, conditions roughly equivalent to the ideal ones.

The presence of larger dispersion to ground makes the line behavior a bit different from the ideal one, but still capable to give the expected advantages. This justifies the wide recent success of $2 \times 25 \mathrm{kV}$ lines over the $1 \times 25$ counterpart.

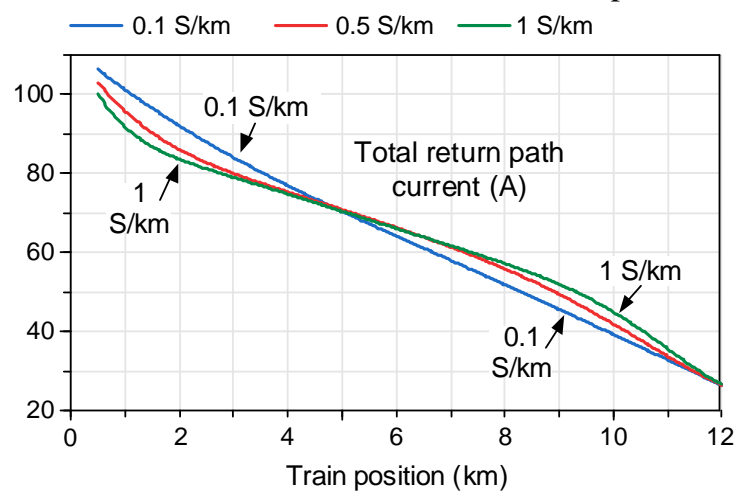

Figure 10. Sum of the three return conductor currents ( $\mathrm{Ra}, \mathrm{Rb}$, , $\mathrm{RW}$ ) as a function of the distributed conductance $\mathrm{g}$.

\section{Ground return and Carson's model}

Carson himself in his original paper [1] suggested reasonable truncation of his infinite-series evaluation of parameters involving ground return. Nowadays, automatic evaluation of many terms is not an issue, in particular considering that this has to be done just once, at the beginning on a time-domain simulation, to evaluate parameters of a given geometry. For all the plots of this paper, referring to 50 $\mathrm{Hz}$, the formulas shown in Appendix A are used, which are by far accurate enough for this frequency. 
Further analysis of Carson's infinite-series expansion and approximation can be found in [2].

To give a concrete idea of the effect of ground, as modelled by Carson equivalent, the plot of current flowing in the ground for different train positions, measured always at its maximum (i.e. midway between feeding station and train position) is shown in figure 11, where it is compared to the current in the return wires.

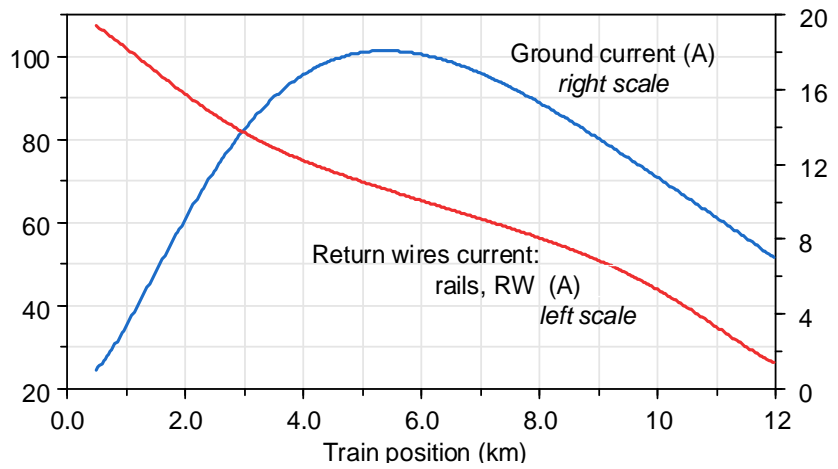

Figure 11. Current (RMS) flowing in ground and return wires.

\section{E. Discussion and Validation}

The results shown are consequences of the basic model hypotheses: MTL transmission line with Carson's model of ground and distributed current rails-to-ground. Their validity comes from the validity of the hypotheses, and of the combined usage proposed here.

The MTL model $(2,3)$ has been widely used in railway lines and experimentally validated, at large frequency intervals in $[13,20]$. Carson model for ground return is 91 year-old and has been continuously used and validated. Its limits of usage have also been widely discussed in literature $[4,5,8])$. Finally, usage of distributed conductance for railsto-soil current dispersion is also a common choice and used for instance in $[7,10]$.

Despite of this validation of the proposed model's individual components, its global validity must not taken for granted without discussion. In particular the use of distributed conductance to ground in conjunction with Carson's ground model is the combination of two techniques independently developed and therefore the accuracy of their combination is not guaranteed.

Consequently, experimental verification is rather necessary. Unfortunately, making the needed measures is very difficult, time-consuming and expensive, and very little literature exists providing measurement results (cf. [11]).

In case sufficient measurement results are published, especially regarding rails' voltages, the author will write a specific paper on fine-tuning and validation of this tool against them.

The results of this paper, for what regards currents, have been checked with another simulation program, developed years ago and successfully used for many years, thus accurate and reliable even though not very flexible and not adequate for easy simulation of moving trains. This (successful) comparison is reported in $[25,26]$.

\section{CONCLUSIONS}

The paper has discussed several issues that must be dealt with when analyzing the problem of AC railway supply system modelling.

The approach followed moves from the conventional analysis of power lines to which it adds, in a coherent way, the complexity connected with ground return, in particular effect of soil (according to Carson's theory) and of distributed conduction towards soil.

All the formulas and techniques proposed are applied in a significant case study, and the results are shown, which are coherent to what known in literature; in particular the issues connected with modelling earth return are analyzed in detail: distributed conductance and soil modelling. The effect of distributed rail-to-soil currents have been evaluated, with special reference to the rails voltage, and the corresponding safety issues discussed. The need of further validating this voltage has been stated and discussed.

Since all the models are created using Modelica simulation language, which is particularly suited for dynamic system modelling, they can be easily incorporated in models including simulation of train movement.

A separate paper will discuss Modelica implementation details [28].

\section{APPENDIX A: ELEMENTS OF $\boldsymbol{Z}$ ’AND $\boldsymbol{Y}^{\prime}$ MATRICES}

\section{A. Z' matrices}

Longitudinal impedances as computed using Carson' theory can be conveniently expressed using auxiliary parameter $P_{\mathrm{ij}}$ and $Q_{\mathrm{ij}}$ (symbols that were used by Carson himself).

The paper uses approximations, valid for $a_{i j} \leq 0.25$, where $a_{i j}=D_{i j} \sqrt{\omega \sigma \mu}$ and

$\omega$ is the radian frequency of voltages and currents,

$\sigma$ is the ground conductivity,

$\mu$ is the ground magnetic permeability.

$D_{i j}$ is twice the conductor height over ground for self inductance, and the distance between a conductor and the symmetrical image of the other image (the soil surface being the symmetry plane), for mutual impedances.

According to the condition $a_{i j} \leq 0.25$ it is:

$P_{i j}=\frac{\pi}{8}-\frac{\sqrt{2}}{6} a_{i j} \cos \theta_{i j}+\frac{a_{i j}^{2}}{16} \cos 2 \theta_{i j} \times\left(0.6728+\ln \frac{2}{a_{i j}}\right)$

$$
+\frac{a_{i j}^{2}}{16} \theta_{i j} \cdot \sin 2 \theta_{i j}
$$

$Q_{i j}=-0.03861+\frac{1}{2} \ln \frac{2}{a_{i j}}+\frac{\sqrt{2}}{6} a_{i j} \cos \theta_{i j}$

In these formulas $\theta_{i j}$ is the angle between the ideal line joining conductor $j$ and the image of conductor $j$, and a vertical straight line. When $i=0$, therefore, $\theta_{i j}=0$.

Based on $P_{\mathrm{ij}}$ and $Q_{\mathrm{ij}}$, the longitudinal self-impedances of conductor $i$ will be:

$Z_{i}^{\prime}=\left(R_{i}+\Delta R_{i}^{\prime}\right)+j \omega\left(\frac{\mu_{0}}{2 \pi} \ln \frac{2 h_{i}}{k_{s i} r_{i}}+\Delta X^{\prime}{ }_{i}\right)$

Where $R_{\mathrm{i}}$ is the conductor's resistance, $r_{\mathrm{i}}$ is the conductor's radius $k_{\mathrm{si}}$ is a factor taking into account the conductor's internal 
reactance ${ }^{1}, h_{\mathrm{i}}$ is the vertical distance between the considered conductor and ground and

$$
\begin{gathered}
\Delta R_{i}^{\prime}=4 \omega P_{i i} \frac{\mu}{4 \pi}=4 \omega P_{i i} \times 10^{-7}, \\
\Delta X^{\prime}{ }_{i}=4 \omega Q_{i i} \frac{\mu}{4 \pi}=4 \omega Q_{i i} \times 10^{-7}
\end{gathered}
$$

are the corrective factors due to the soil. Mutual impedances are:

$Z_{i j}^{\prime}=\Delta R^{\prime}{ }_{i j}+j \omega\left(\frac{\mu_{0}}{2 \pi} \ln \frac{D_{i j}}{d_{i j}}+\Delta X^{\prime}{ }_{i j}\right)$

Where $D_{i j}$ was already discussed and $d_{i j}$ is the distance between conductor $i$ and $j$.

\section{B. Y' matrices}

Regarding matrix $\boldsymbol{Y}^{\prime}$, it is $\boldsymbol{Y}^{\boldsymbol{\prime}}=\boldsymbol{P}^{\boldsymbol{\prime}-1}$ where the elements or matrix $\boldsymbol{P}^{\prime}$ are:

$p_{i i}^{\prime}=\frac{1}{2 \pi \epsilon} \ln \frac{2 h_{i}}{r_{i}} \quad p_{i j}^{\prime}=\frac{1}{2 \pi \epsilon} \ln \frac{D_{i j}}{d_{i j}} \quad($ this when $j \neq j$ )

So, computation of $\boldsymbol{Y}^{\prime}$ requires a matrix inversion. Note that $p^{\prime}{ }_{\text {ij }}$ are totally different quantities from the $P_{\mathrm{ij}}$ used above.

\section{APPENDIX B: STRUCTURE OF THE CASE-STUDY LINE}

The architecture of a typical $2 \times 25 \mathrm{kV}$ system can be represented as in figure $\mathrm{A} 1$

Only one track is shown, while the other track is connected to the same transformers and autotransformers as indicated by the dashed lines in figure.

The HV source feeds, by means of two single-phase transformers, the first cell of the line. The single-phase transformers are both connected to the same two phases of the HC source. The transformers feed basically three conductors: the upper conductor, constituted by messenger and contact wire, the central conductor, constituted by rails, and additional ground wires, all connected to each other, and the so-called feeder. How these conductors are physically located is shown in figure 1 in the main body of the paper.

After some length of line (in Italy $12.5 \mathrm{~km}$ ), the so-called first cell ends; the autotransformer at the final end of the first cell is a single winding on an iron core, with a connection at its half; therefore the number of turns of its upper and lower parts are equal to each other. After the first cell, a second cell is present, fed at its beginning and ending by autotransformers, whose length is equal to the first cell.

The disposition of feeder and contact wire is as per figure 1 , of which only a single track is considered.

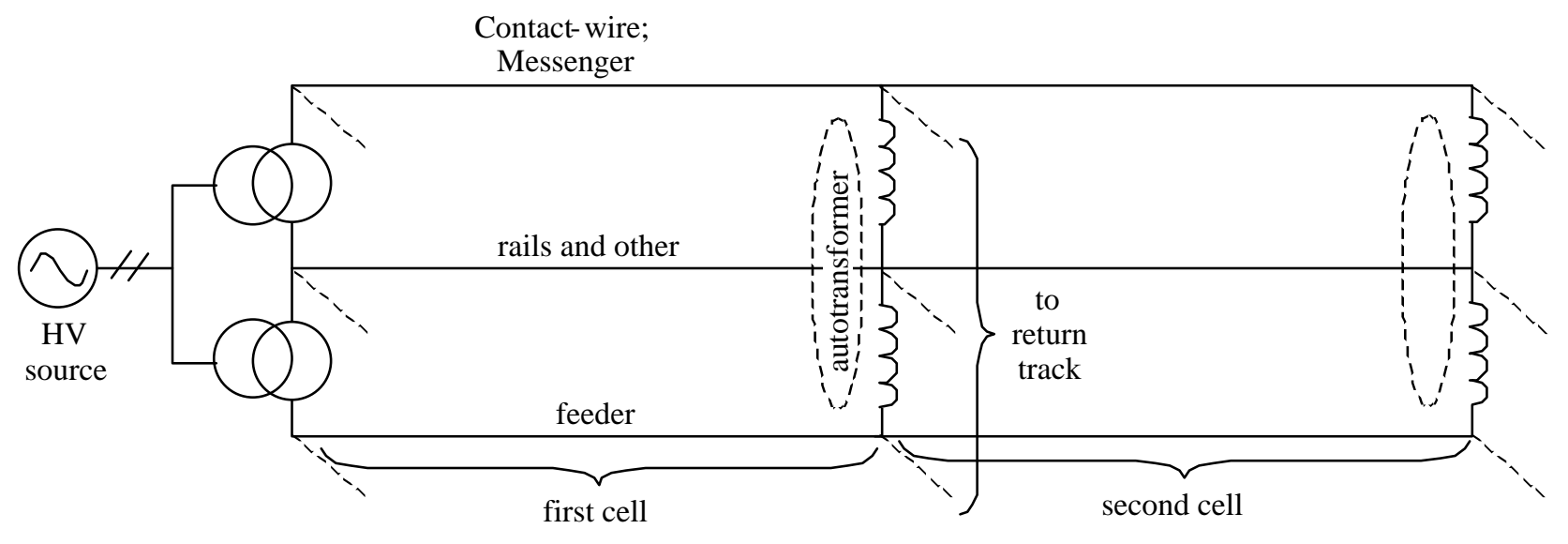

Figure A1: A typical architecture of a $2 \times 25 \mathrm{kV}$ supply system, which is used for the case study of the paper.

\section{REFERENCES}

[1] J. R. Carson, "Wave Propagation in Overhead Wires with Ground Return", The Bell System Technical Journal, 1926, Vol. 5 , issue 4;

[2] FF. Alvarado, R. Betancourt, "An accurate closed-form approximation for ground return impedance calculations", Proceedings of the IEEE, vol.71, No.2, February 1983, pp.279280.

[3] R. J. Hill, C. Carpenter, "Modelling of nonlinear rail impedance in AC traction power systems", IEEE Trans. on Power Delivery, vol. 6, n. 4, October 1991;

[4] J. Hill, D. Carpenter, "Rail Track Distributed Transmission Line Impedance and Admittance: Theoretical Modeling and

\footnotetext{
${ }^{1} k_{\mathrm{s}}$ for non-stranded, dielectric conductors is equal to 0.778 ; for stranded dielectric conductors is between 0.70 and 0.778 ; since
}

Experimental results", IEEE Trans. on Vehicular Technology, vol. 42, n. 2, May 1993.

[5] M. D'Amore, M.S. Sarto, "Simulation Models of a Dissipative Transmission Line Above a Lossy ground for a Wide-Frequency Range - Part II: Multiconductor Configuration", IEEE Trans. on Electromagnetic Compatibility, Vol 38, May 1996.

[6] R. J. Hill, S. Briante, P. J. Leonard, "Railway track transmission line parameters from finite element field modelling: Series impedance", IEEE Proc. on Electric Power Applications, vol. 146, n. 6, Nov. 1999.

[7] R. J. Hill, S. Brillante, P. J. Leonard, "Railway track transmission line parameters from finite element field modelling: Shunt admittance", IEE Proc.-Electr. Power Appl.. Vol. 147, No. 3, May 2000

formulas are not strongly dependent on this number, a generic value can be used, e.g., 0.75 . 
[8] F. A. Uribe, J. L. Naredo, P. Moreno, L. Guardado, "Calculating Earth Impedances Of Underground Transmission Cables", Proc. of Int, Conf. on Power System Transients, IPST Rio de Janeiro, Brazil, 2003

[9] R. Marconato: Electric Power Systems, Volume 1. Background and Basic components, Second Edition, ISBN 88-432-0014-3

[10] A. Mariscotti, P. Pozzobon, "Determination of the Electrical Parameters of Railway Traction Lines: Calculation, Measurement, and Reference Data", IEEE Trans. on Power delivery, vol. 19, n. 4, Oct 2004, pp 1538-1546.

[11] R. Cella, et al: "Measurement of AT Electric Railway System Currents at Power-Supply Frequency and Validation of a Multiconductor Transmission-Line Model”, IEEE transactions on Power delivery, Vol 21. N. 3, July 2006, pp. 1721-1726.

[12] G. Lucca: "Propagazione di tensione e corrente lungo un binario: due modelli di calcolo a confronto", Ingegneria Ferroviaria, Feb. 2005.

[13] C. R. Paul, “Analysis of Multiconductor Transmission Lines”, book, IEEE/Wiley, 2008 ISBN 978-0-470-13154-1, pp 282342.

[14] A. Capasso, M. Ciucciarelli, S. Lauria: "An Integrated methodology cor $2 \times 25 \mathrm{kV}, 50 \mathrm{~Hz}$ traction system calculation". Ingegneria Ferroviaria, Nov. 2009, pp. 979-998.

[15] P. Fritzson, P. Aronsson, A. Pop, H. Lundvall, K. Nystrom, L. Saldamli. D. Broman, "OpenModelica - a free open-source environment for system modeling, simulation, and teaching" Digital Object Identifier: 10.1109/CACSD-CCA-ISIC.2006. 4776878. Publication year: 2006, page(s) 1588-1595

[16] P. Fritzson, "Introduction to Modeling and Simulation of Technical and Physical Systems with Modelica", Wiley-IEEE Press ISBN:978-1-118-01068-6, September 2011.

[17] M. Ceraolo and D: Poli: "Fundamentals of Electric Power Engineering”, IEEE/Wiley 2014, ISBN 978-1-118-67969-2

[18] Peter Fritzson, "Principles of Object-Oriented Modeling and Simulation with Modelica 3.3: A cyber-phywsical approach" Wiley-IEEE Press SBN: 978-1-118-85912-4, 2015.

[19] A Ametani, et al., "A Study on High-Frequency Wave Propagation along Overhead Conductors by Earth-return Admittance/Impedance and Numerical Electromagnetic Analysis", Proc. of Int, Conf. on Power System Transients, IPST, Cavtat, Croatia 2015.

[20] A. Dolara, M. Gualdoni, S. Leva, "Reduced Multiconductor Transmission Line models for Power Quality Analysis in Railway Systems", $15^{\text {th }}$ IEEE Int. Conf. on Harmonics and Qual. of Power, ICHQP, Hong Kong, 2012.

[21] N. Noroozi et al, "Fault analysis on AC railway supply system", $6^{\text {th }}$ IEEE PEDST, Theaan, Iran, Feb. 2015, pp 567-572.

[22] A. Capasso, M. Ceraolo, R. Lamedica, G. Lutzemberger, A. Ruvio, "Modelling and simulation of electric urban transportation systems with energy storage", in Proc. $16^{\text {th }}$ IEEE Intern. Conf. on Environment and Electrical Engineering (EEEIC), IEEE, Florence 7-10 June 2016.

[23] G. Aloisio, P. Bolognesi, A. Capasso, M. Ceraolo, R. Lamedica, G. Lutzemberger, A.Ruvio, "Modelling $2 \times 25 \mathrm{kV}-50 \mathrm{~Hz}$ traction systems for power frequency studies", IEEE Int. Conf. on Environment and Electrical Engineering, Milan, Italy, June 2017.

[24] INECO Comparative study of the Electrification Systems 1x25 $\mathrm{kV}$ and $2 \times 25 \mathrm{kV}$, Madrid June 2011, available on September 2017) https://www.scribd.com/document/249040681/INECOComparative-Study-1x25-2x25 (retrieved on September 2017)

[25] G. Aloisio, P. Bolognesi, A. Capasso, M. Ceraolo, R. Lamedica, G. Lutzemberger, A. Ruvio, L. Sani, "Modelling $2 \times 25$ kV -50 $\mathrm{Hz}$ traction systems for power frequency studies", $17^{\text {th }}$ International Conference on Environment and Electrical Engineering (EEEIC), 2017 IEEE, 6-9 June 2017, Milan, Italy

[26] A. Capasso, R. Lamedica, A. Ruvio, M. Ceraolo, G. Lutzemberger: "New approaches to simulate AC electrified railay Modelling and simulation of $2 \times 25 \mathrm{kV}-50 \mathrm{~Hz}$ complete system" AEIT 2017, International Annyual Conference, 20/22 September 2017, Cagliari, Italy.

[27] https://www.modelica.org
[28] M. Ceraolo: "Long duration simulations of railway AC Electrified lines", submitted for publication to IET Electrical Systems in Transportation. 